\title{
Experiments with photons at MAMI
}

\author{
R. Beck ${ }^{\mathrm{a}}$ \\ Helmholtz-Institut für Kern- und Strahlenphysik, Nussallee 14-16, 53115 Bonn, Germany \\ / \\ Published online: 26 May 2006 - C Società Italiana di Fisica / Springer-Verlag 2006
}

\begin{abstract}
A very successful experimental program with real photons has been achieved in 20 years of operation at the Mainz Microtron (MAMI) facility. The different detector setups, like DAPHNE, TAPS and the Crystal Ball are centered around the tagged photon facility the so-called Glasgow Tagger. From the rich spectrum of results only a few highlights will be discussed here, the proton polarizabilities, the pion polarizabilities, pion photoproduction close to the pion threshold and in the $\Delta(1232)$-resonance region.
\end{abstract}

PACS. 13.40.-f Electromagnetic processes and properties - 13.60.Le Meson production - 13.60.Fz Elastic and Compton scattering - 14.20.Dh Protons and neutrons

\section{Introduction}

Experiments with real photons at MAMI have been performed in the framework of the A2-collaboration. Monochromatic photons from bremsstrahlung tagging by the Glasgow Tagger $[1,2]$ are used for all experiments. Polarized photon beams, linear and circular, are available as well as polarized targets.

Groups from several institutions and countries (see [3]) have provided different detector components for example the photon spectrometer TAPS [4], the $4 \pi$ charge particle tracking detector DAPHNE [5] and more recent the photon spectrometer Crystal Ball [6]. Many data have been taken on the proton and on light and complex nuclei including the total photon absorption, Compton scattering, meson production, break up reactions and multi pion production in the final state. The experimental work was based on 175 Diploma — and PhD - thesis, which are published in more than 100 refereed articles. Here only a few highlights of this experimental program can be adressed.

\section{Low-energy Compton scattering}

Next to the size and the anomalous magnetic moment, the polarizability is a further property of a particle with a substructure. In the present of the electromagnetic fields $(E$ and $B)$, electric dipole moments are induced and magnetic dipole moments may be oriented (paramagnetism) or induced according to Lenz's rule (diamagnetism). The most precise determination of the proton polarizabilities comes from Compton scattering experiments. These measurements rely on a Low-Energy Theorem to establish a

\footnotetext{
a e-mail: beck@hiskp.uni-bonn.de
}

unique relation between a low-energy expansion of the Compton-scattering cross section and the static polarizabilities $\alpha$ and $\beta$. For photon energies small compared to pion mass, this expansion reads $[7,8]$ :

$$
\begin{aligned}
\left(\frac{\mathrm{d} \sigma}{\mathrm{d} \Omega}\right) & =\left(\frac{\mathrm{d} \sigma}{\mathrm{d} \Omega}\right)_{\text {Point }} \\
& -\omega \omega^{\prime}\left(\frac{\omega^{\prime}}{\omega}\right) \frac{e^{2}}{m}\left[\frac{\alpha+\beta}{2}(1+z)^{2}+\frac{\alpha-\beta}{2}(1-z)^{2}\right]
\end{aligned}
$$

with $z=\cos \left(\theta_{\gamma}\right)$, where $\omega$ and $\omega^{\prime}$ are the energies of the incident and scattered photon, respectively; $\frac{\mathrm{d} \sigma}{\mathrm{d} \Omega}$ Point is the exact cross section for a structureless proton with an anomalous magnetic moment. The quantities $\alpha$ and $\beta$ are the static polarizabilities.

Low-energy Compton scattering from the proton in the energy range from $55 \mathrm{MeV}$ to $165 \mathrm{MeV}$ was measured using the TAPS detector set up at the photon beam at MAMI. The energy of the incident electron beam was chosen to be $180 \mathrm{MeV}$. The target consisted of a Kapton cylinder of $20 \mathrm{~cm}$ length filled with liquid hydrogen. Data obtained from about $200 \mathrm{~h}$ of beam time were analysed [9, 10]. The scattered photons were detected with 6 blocks of the TAPS. Since the recoiling protons could not be detected, a single-particle trigger had to be used. Therefore, this minimum bias trigger included all kinds of background events for example cosmic ray events which have not been suppressed by an active shield and electromagnetic background from the beam collimation system and from the target itself. These sources of background were partially suppressed by time cuts and a missing-energy cut, which is defined as the difference between the measured incident photon energy (tagger) and the expected incident photon, as calculated from the measured scattered photon assuming Compton kinematics. 


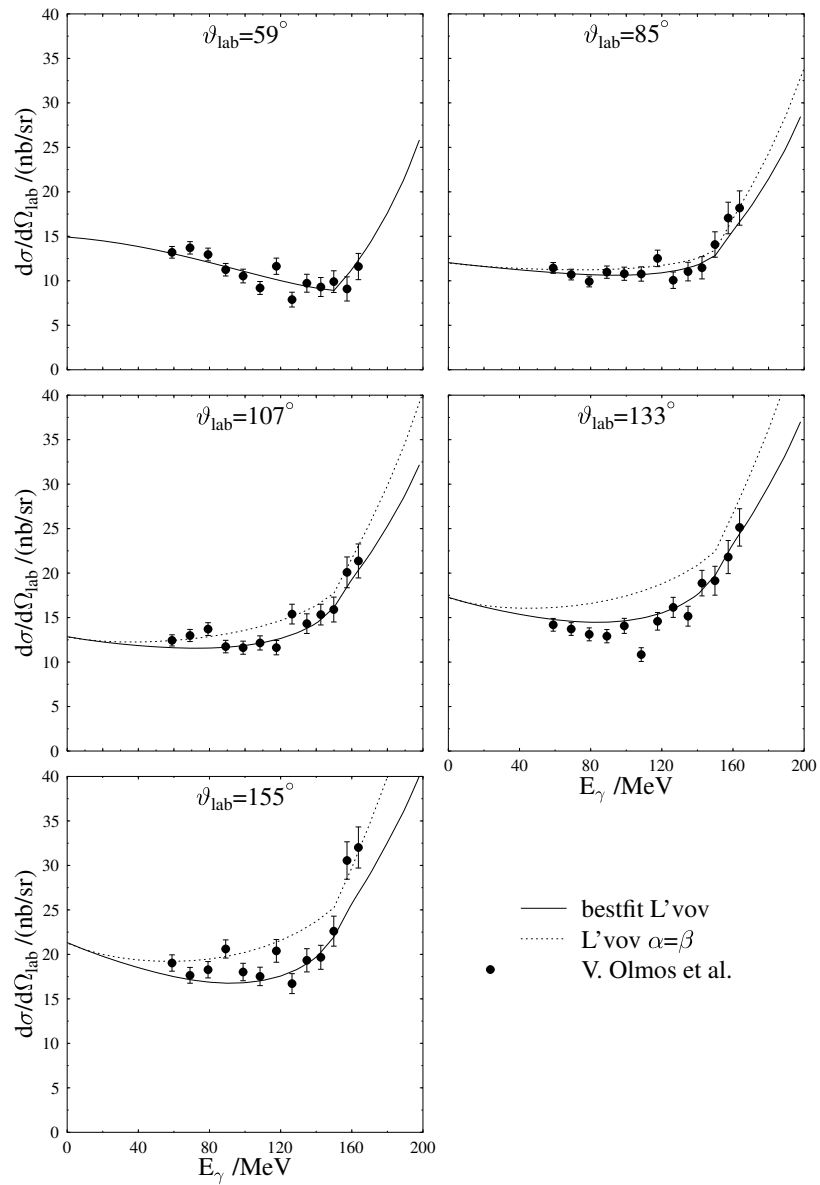

Fig. 1. Measusred differential cross-sections in the lab system [9] compared with a dispersion relation calculation (solid line) [11].

The differential cross sections obtained are plotted in fig. 1. The systematic errors of $\pm 3 \%$ arise from uncertainties in the photon flux $( \pm 2 \%)$ and the target density $( \pm 2 \%)$ combined in quadrature. The effective solid angles were determined with Monte Carlo simulations. Errors from uncertainties in the experiment geometry are estimated to be $\pm 5 \%$. With the help of the dispersion relation approach the electromagnetic polarizabilities of the proton can be extracted from the experimental cross-sections. The procedure used in the analysis was to take $\alpha$ and $\beta$ as free parameters, and sometimes as well the constraint given by the Baldin sum rule. Using standard $\chi^{2}$ minimization, the result obtained, when fitting the MAMI/TAPS data alone without the sum rule constraint, is

$$
\begin{aligned}
& \alpha=11.9 \pm 0.5(\text { stat. }) \mp 1.3(\text { syst. }), \\
& \beta=1.2 \pm 0.7(\text { stat. }) \pm 0.3(\text { syst. }) .
\end{aligned}
$$

The Baldin sum rule obtained from this result, $\alpha+\beta=13.1 \pm 0.9$ is in agreement with the value determined by the total photon absorption cross section.

A fit to the existing low-energy Compton scattering data including the new MAMI/TAPS data and the sum

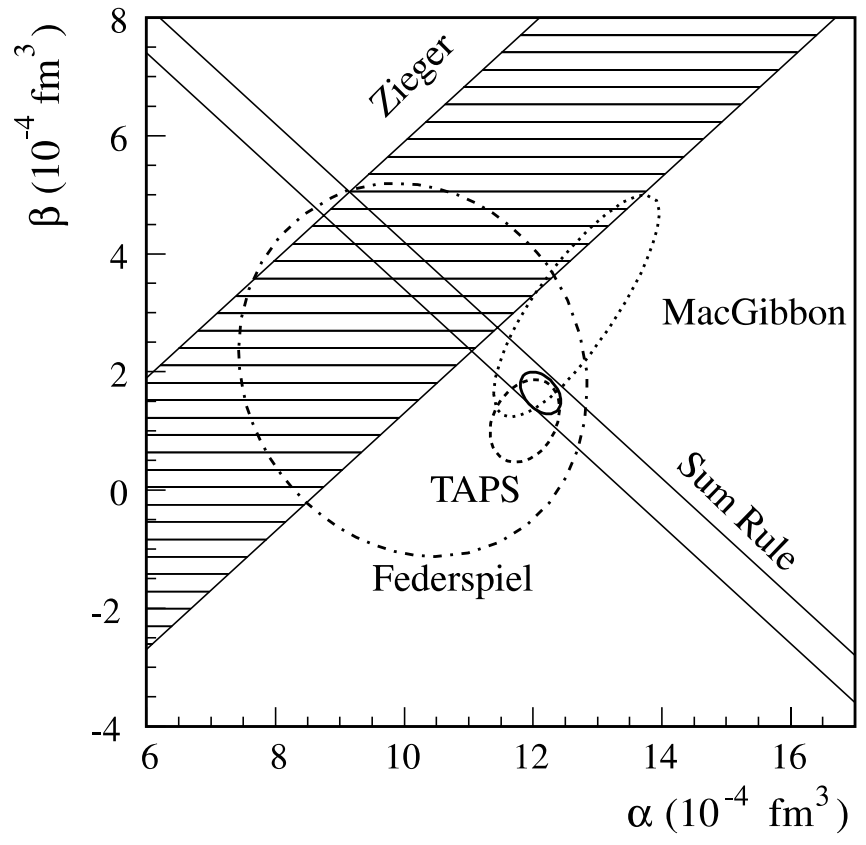

Fig. 2. Error contour plot in the $(\alpha-\beta)$-plane for which only the statistical errors are taken. The contours correspond to the values $\chi_{\min }^{2}+1$ of the individual fits. Also shown are the sum rule constraint and the value $\alpha-\beta$ as follows from the experiment by Zieger et al. [12]. The thick solid line shows the result of the global fit, eq. (4).

rule constraint leads to the following result:

$$
\begin{aligned}
& \alpha=12.1 \pm 0.3_{\text {stat. }} \mp 0.4_{\text {syst. }} \pm 0.3_{\text {mod. }} \\
& \beta=1.6 \pm 0.4_{\text {stat. }} \pm 0.4_{\text {syst. }} \pm 0.4_{\text {mod. }}
\end{aligned}
$$

where the first error denotes the statistical, the second the systematic and the third the model-dependent one. The results are summarized in fig. 2 (contourplot) where the contours in the $(\alpha-\beta)$ plane for $\chi_{\min }^{2}+1$ are plotted. In addition, the Baldin sum and the value obtained from the experiment by Zieger [12] are included.

\section{Pion polarizability}

The pion polarizabilities characterize the dynamical deformation of the pion in the electromagnetic field. The values of the electric $\alpha$ and magnetic $\beta$ pion polarizabilities depend on the rigidity as a composite particle and provide important information of internal structure. Very different values for the pion polarizabilities have been calculated in the past. All predictions agree, however, that the sum of the two polarizabilities of the $\pi^{ \pm}$meson is very small. On the other hand, the values of the difference of the polarizabilities are very sensitive to theoretical models. For example, investigations within the framework of the chiral perturbation theory $(C h P T)$ predict $(\alpha-\beta)_{\pi^{ \pm}} \approx 5.4[13]$ in one-loop calculations and $4.4 \pm 1.0$ for two-loops [14] (all values of the polarizabilities are given in units of $10^{-4} \mathrm{fm}^{3}$ ). The calculations in the extended Nambu-Jona 

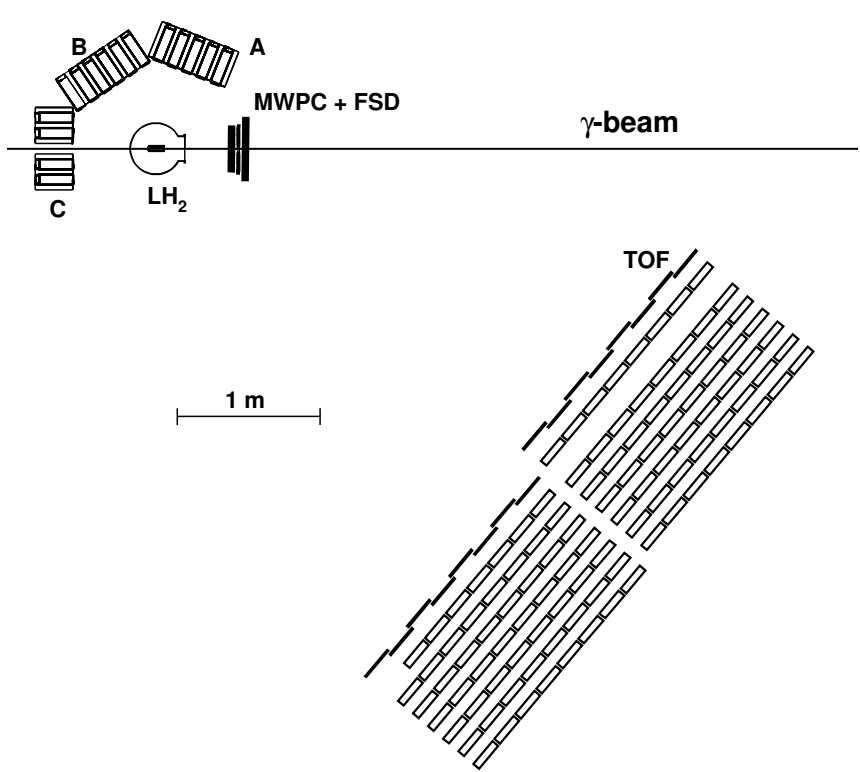

Fig. 3. Floor plan of the experimental setup showing the location of the detectors. A, B, C are TAPS blocks, MWPC+FSD show multi-wire proportional chambers and the forward scintillation detector, TOF indicates the block of the neutron detector bars, and $\mathrm{LH}_{2}$ stands for the liquid-hydrogen target in its vacuum scattering chamber.

Lasinio model with linear realization of chiral $U(3) \times U(3)$ symmetry [15] result in $\alpha_{\pi^{ \pm}}=-\beta_{\pi^{ \pm}}=3.0 \pm 0.6$. The application of dispersion sum rules (DSR) at fixed value of the Mandelstam variable $u=\mu^{2}$ for calculation of this parameter $[16,17]$ leads to $(\alpha-\beta)_{\pi^{ \pm}}=10.3 \pm 1.9$. DSR at finite energy [8] gave the similar result: $(\alpha-\beta)_{\pi^{ \pm}}=10.6$. A calculation in the linear $\sigma$ model with quarks and vector mesons included to one loop order predicted $(\alpha-\beta)_{\pi^{ \pm}}=$ 20 [18]. An evaluation in the Dubna quark confinement model [19] results in $(\alpha-\beta)_{\pi^{ \pm}}=7.05$.

Because there is no stable pion target, experimental information about the pion polarizabilities is not easy to obtain. One has to investigate reaction channels, like scattering high energy pion in the Coulomb field of a heavy nuclei or the radiative pion photoproduction.

The scattering of high energy pions off the Coulomb field of heavy nuclei [20] has resulted in $\alpha_{\pi^{-}}=-\beta_{\pi^{-}}=$ $6.8 \pm 1.4 \pm 1.2$. This value agrees with prediction of the dispersion sum rules but is about 2.5 times larger than the ChPT result. The experiment of the Lebedev Institute on radiative pion photoproduction from the proton [21] has given $\alpha_{\pi^{+}}=20 \pm 12$. This value has large error bars and shows the largest discrepancy with regard to the ChPT predictions. The attempts to determine the polarizability from the reaction $\gamma \gamma \rightarrow \pi \pi$ suffer greatly from theoretical [22] and experimental [23] uncertainties. The most recent analysis of MARK II and Crystal Ball data [24] finds no evidence for a violation of the ChPT predictions. However, even changes of polarizabilities by $100 \%$ and more are still compatible with the present error bars.

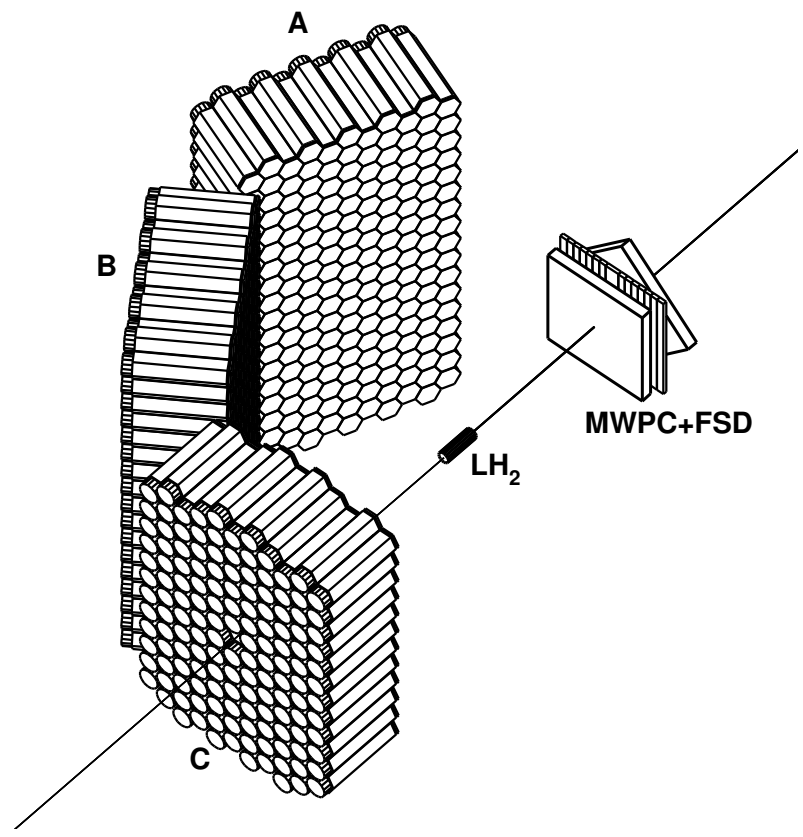

Fig. 4. Enlarged view showing the details of the TAPS configuration.

The experiment discussed here has been performed at the continuous-wave electron accelerator MAMI B [25, 26] using Glasgow-Edinburgh-Mainz tagger photon facility $[1,2]$. The quasi-monochromatic photon beam covered the energy range from 537 to $819 \mathrm{MeV}$ with an intensity $\sim 6 \times 10^{5} / \mathrm{s}$ in the tagger channel for the lowest photon energy and average energy resolution of $2 \mathrm{MeV}$. The tagged photons entered a scattering chamber, containing a $3 \mathrm{~cm}$ diameter and $11.4 \mathrm{~cm}$ long liquid hydrogen target with Capton windows. The emitted photon $\gamma^{\prime}, \pi^{+}$meson, and the neutron were detected in coincidence. The experimental setup is shown in fig. 3 .

The photons were detected by the spectrometer TAPS [4], assembled in a special configuration (fig. 4). The TAPS spectrometer consists of $528 \mathrm{BaF}_{2}$ crystals. Each hexagonally shaped crystal is $250 \mathrm{~mm}$ long corresponding to 12 radiation lengths. All crystals were arranged into three big blocks. Two blocks (A, B) consisted of 192 crystals arranged in 11 columns and the third block $(\mathrm{C})$ had 144 crystals arranged in 11 columns. These three blocks were located in the horizontal plane around the target at angles $68^{\circ}, 124^{\circ}, 180^{\circ}$ with respect to the beam axis. Their distances to the target center were $55 \mathrm{~cm}, 50 \mathrm{~cm}$ and $55 \mathrm{~cm}$, respectively. All $\mathrm{BaF}_{2}$ modules were equipped with $5 \mathrm{~mm}$ thick plastic veto detectors for the identification of charged particles.

The neutrons were detected by a wide aperture timeof-flight spectrometer (TOF) [27]. It consisted of 111 scintillation detector bars of $50 \times 200 \times 3000 \mathrm{~mm}^{3}$ and 16 counters $\left(10 \times 230 \times 3000 \mathrm{~mm}^{3}\right)$ which were used as veto detectors. The bars are made from NE110 plastic scintilator and each bar is read out on both ends by two $3^{\prime \prime}$ phototubes XP2312B. All bars were assembled in 8 planes 


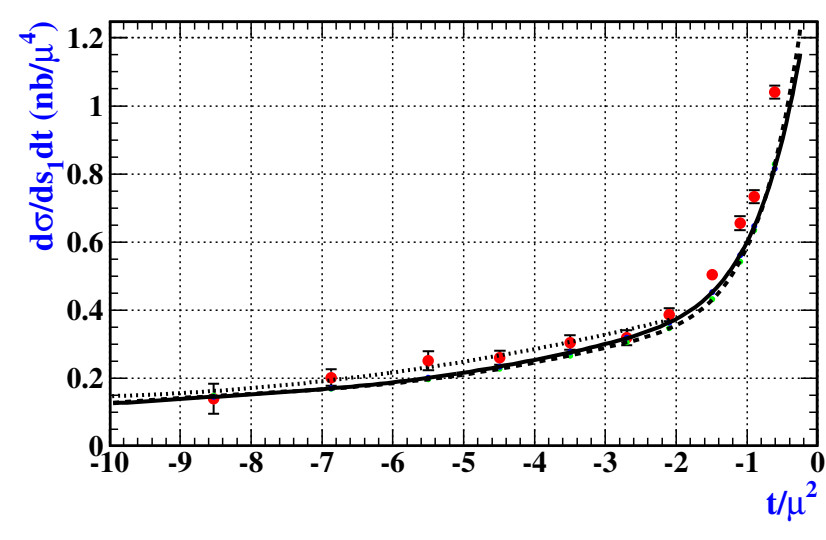

Fig. 5. The differential cross section of the process $\gamma p \rightarrow \gamma \pi^{+} n$ averaged over the full photon beam energy interval and over $s_{1}$ from $1.5 m_{\pi}^{2}$ to $5 m_{\pi}^{2}$. The solid and dashed lines are the predictions of model- 1 and model-2, respectively, for $(\alpha-\beta)_{\pi^{+}}=0$. The dotted line is a fit to the experimental data (see text).

of a special configuration with 16 detectors in each, following one after another (fig. 3). Such a neutron detector allows to detect the neutrons in the energy region 10 $100 \mathrm{MeV}$ with efficiency $30-50 \%$ and to determine their energy with a resolution $\sim 10 \%$ using the neutron time of flight and the angle of the neutron emission measured with a precision $\sim 2-3 \%$.

To detect the $\pi^{+}$meson two two-coordinate multiwire proportional chambers (MWPC) and a forward scintillator detector (FSD), for getting a fast trigger signal, have been developed and constructed. The MWPC's overlap angles in the laboratory system were $\theta \cong 2^{\circ}-20^{\circ}$, $\varphi \cong 0^{\circ}-360^{\circ}$ and were located under $0^{\circ}$ with respect to the beam direction.

The cross section of the process $\gamma p \rightarrow \gamma \pi^{+} n$ has been calculated in the framework of two different models. In the first model (model-1) the contribution of all the pion and nucleon pole diagrams is taken into account using pseudoscalar pion-nucleon coupling [28].

In the second model (model-2), the nucleon and the pion pole diagrams without the anomalous magnetic moments of the nucleons, and in addition the contributions of the resonances $\Delta(1232), P_{11}(1440), D_{13}(1520)$, and $S_{11}(1535)$ are included.

To control the model dependence of the result the kinematic regions were limited to regions where the difference between model-1 and model-2 does not exceed $3 \%$ when $(\alpha-\beta)_{\pi^{+}}$is constrained to zero. First, a kinematic region where the contribution of the pion polarizability is negligible, i.e. the region $1.5 m_{\pi}^{2} \leq s_{1}<5 m_{\pi}^{2}$ was analysed, where $s_{1}$ is the squared pion-photon center-of-mass energy.

In fig. 5, the experimental data for the differential cross section, averaged over the full photon beam energy interval from $537 \mathrm{MeV}$ up to $817 \mathrm{MeV}$ and over $s_{1}$ in the indicated interval, are compared to predictions of model1 (dashed curve) and model-2 (solid curve). The dotted curve is the fit of the experimental data in the region of $-10 m_{\pi}^{2}<t<-2 m_{\pi}^{2}$, where $t$ is the squared pion mo-

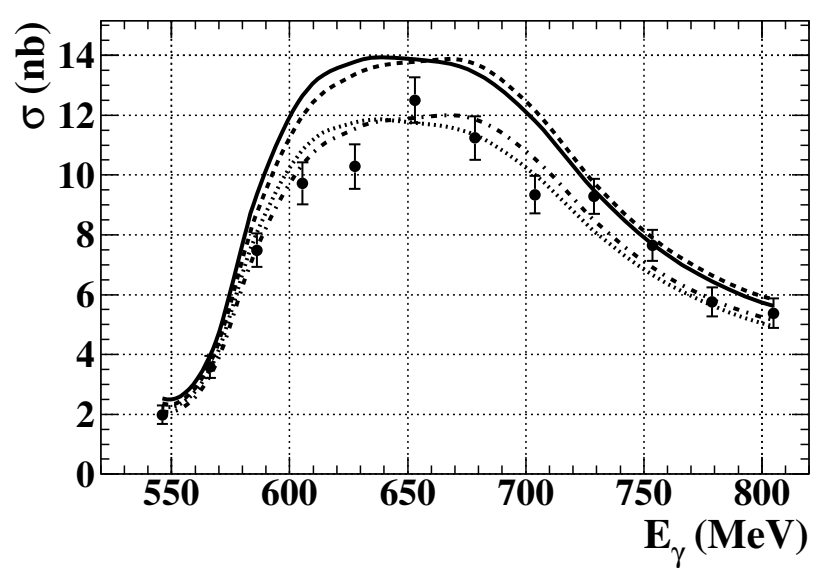

Fig. 6. The cross section of the process $\gamma p \rightarrow \gamma \pi^{+} n$ integrated over $s_{1}$ and $t$ in the region where the contribution of the pion polarizability is biggest and the difference between the predictions of the theoretical models under consideration does not exceed 3\%. The dashed and dashed-dotted lines are predictions of model- 1 and the solid and dotted lines of model-2 for $(\alpha-\beta)_{\pi^{+}}=0$ and $14 \times 10^{-4} \mathrm{fm}^{3}$, respectively.

mentum transfer. As seen from this figure, the theoretical curves are very close to the experimental data. This means that the dependence of the differential cross section on the square of the four-momentum transfer $t$ which is basically the kinetic energy of the neutron is well reproduced by using the mentioned GEANT simulations for the efficiency.

In a second step, the kinematic region where the polarizability contribution is maximal was investigated. This is the region $5 m_{\pi}^{2} \leq s_{1}<15 m_{\pi}^{2}$ and $-12 m_{\pi}^{2}<t<-2 m_{\pi}^{2}$. In the considered region of the phase space, the cross sections of the process $\gamma p \rightarrow \gamma \pi^{+} n$ integrated over $s_{1}$ and $t$ are calculated according to model- 1 and model- 2 for two different values of $(\alpha-\beta)$. The obtained experimental cross sections and their theoretical predictions for $(\alpha-\beta)_{\pi^{+}}=0$ and $14 \times 10^{-4} \mathrm{fm}^{3}$ are presented in fig. 6 . The error bars are the quadratic sum of statistical and systematic errors.

For each model, we obtain

$$
\begin{aligned}
(\alpha-\beta)_{\pi^{+}}= & \left(12.2 \pm 1.6_{\text {stat }} \pm 3.3_{\text {syst }}\right) \times 10^{-4} \mathrm{fm}^{3} \\
& (\text { model }-1), \\
(\alpha-\beta)_{\pi^{+}}= & \left(11.1 \pm 1.4_{\text {stat }} \pm 2.8_{\text {syst }}\right) \times 10^{-4} \mathrm{fm}^{3} \\
& (\text { model }-2) .
\end{aligned}
$$

Averaging over the results of the two models, the final result is obtained [29]:

$(\alpha-\beta)_{\pi^{+}}=\left(11.6 \pm 1.5_{\text {stat }} \pm 3.0_{\text {syst }} \pm 0.5_{\text {mod }}\right) \times 10^{-4} \mathrm{fm}^{3}$.

\section{Pion photoproduction in the threshold region}

The photoproduction of pions near threshold has been a topic of considerable experimental and theoretical activities over the past years, ever since the results of the experiments, performed in Saclay [30], Mainz ([31,32]) and 
Saskatoon [33], were at variance with the prediction of a low energy theorem (LET), which was derived in the early 70 s $[34,35]$. Being based on fundamental principles, this LET predicted the value of the $S$-wave threshold amplitude $E_{0+}$ in a power series in $\mu=m_{\pi} / m_{N}$, the ratio of the masses of the pion and nucleon.

The discrepancy could be explained by a calculation in the framework of heavy-baryon chiral perturbation theory (ChPT) [36], which showed that additional contributions due to pion loops in $\mu^{2}$ have to be added to the old LET. Refined calculations within heavy-baryon ChPT [37] led to descriptions of the four relevant amplitudes at threshold by well-defined expansions up to order $p^{4}$ in the $S$-wave amplitude $E_{0+}$ and $p^{3}$ in the $P$-wave combinations $P_{1}$, $P_{2}$ and $P_{3}$, where $p$ denotes any small momentum or pion mass, the expansion parameters in heavy-baryon ChPT. To that order, three low-energy constants (LEC) due to the renormalization counter terms appear, two in the expansion of $E_{0+}$ and an additional LEC $b_{P}$ for $P_{3}$, which have to be fitted to the data or estimated by resonance saturation.

However, two combinations of the $P$-wave amplitudes, $P_{1}$ and $P_{2}$, are free of low-energy constants. Their expansions in $\mu$ converge rather well leading to new LETs for these combinations. Therefore, the $P$-wave LETs offer a significant test of heavy-baryon ChPT. However, for this test the $S$-wave amplitude $E_{0+}$ and the three $P$-wave combinations $P_{1}, P_{2}$ and $P_{3}$ have to be separated. This separation can be achieved by measuring the photon asymmetry using linearly polarized photons, in addition to the measurement of the total and differential cross sections.

The differential cross sections can be expressed in terms of the $S$ - and $P$-wave multipoles, assuming that close to threshold neutral pions are only produced with angular momenta $l_{\pi}$ of zero and one. Due to parity and angular momentum conservation only the $S$-wave amplitude $E_{0+}\left(l_{\pi}=0\right)$ and the $P$-wave amplitudes $M_{1+}$, $M_{1-}$ and $E_{1+}\left(l_{\pi}=1\right)$ can contribute and it is convenient to write the differential cross section and the photon asymmetry in terms of the three $P$-wave combinations $P_{1}=3 E_{1+}+M_{1+}-M_{1-}, P_{2}=3 E_{1+}-M_{1+}+M_{1-}$ and $P_{3}=2 M_{1+}+M_{1-}$. The c.m. differential cross section is

$$
\frac{\mathrm{d} \sigma(\theta)}{\mathrm{d} \Omega}=\frac{q}{k}\left(A+B \cos (\theta)+C \cos ^{2}(\theta)\right),
$$

where $\theta$ is the c.m. polar angle of the pion with respect to the beam direction and $q$ and $k$ denote the c.m. momenta of pion and photon, respectively. The coefficients $A=\left|E_{0+}\right|^{2}+\left|P_{23}\right|^{2}, B=2 \operatorname{Re}\left(E_{0+} P_{1}^{*}\right)$ and $C=\left|P_{1}\right|^{2}-\left|P_{23}\right|^{2}$ are functions of the multipole amplitudes with $P_{23}^{2}=\frac{1}{2}\left(P_{2}^{2}+P_{3}^{2}\right)$. Earlier measurements of the total and differential cross sections already allowed determination of $E_{0+}, P_{1}$ and the combination $P_{23}$.

In order to obtain $E_{0+}$ and all three $P$-waves separately and to test the new LETs of ChPT, it is necessary to measure, in addition to the cross sections, the photon asymmetry $\Sigma$,

$$
\Sigma=\frac{\mathrm{d} \sigma_{\perp}-\mathrm{d} \sigma_{\|}}{\mathrm{d} \sigma_{\perp}+\mathrm{d} \sigma_{\|}},
$$

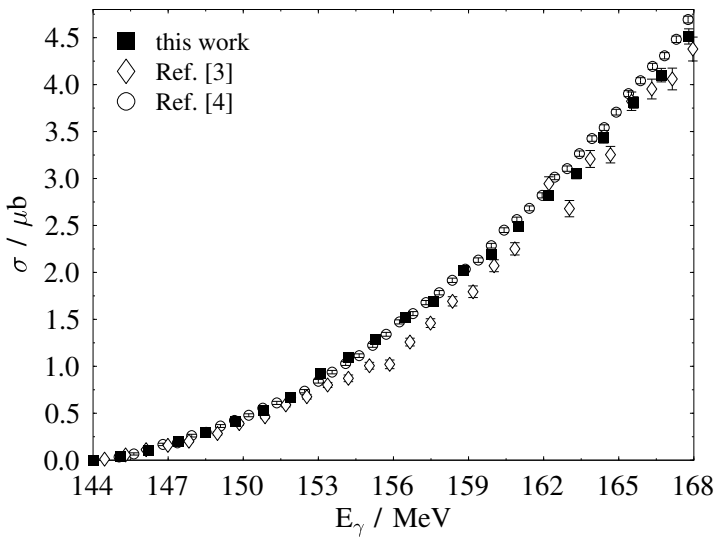

Fig. 7. Total cross sections for $\pi^{0}$ photoproduction close to threshold with statistical errors (without systematic error of $5 \%$ ) as function of incident photon energy (solid squares, this work ref. [38], open circles, ref. [33], open diamonds ref. [32]).

where $\mathrm{d} \sigma_{\perp}$ and $\mathrm{d} \sigma_{\|}$are the differential cross sections for photon polarizations perpendicular and parallel to the reaction plane defined by the pion and proton. The asymmetry is proportional to the difference of the squares of $P_{3}$ and $P_{2}$ :

$$
\Sigma(\theta)=\frac{q}{2 k}\left(P_{3}^{2}-P_{2}^{2}\right) \cdot \sin ^{2}(\theta) / \frac{\mathrm{d} \sigma(\theta)}{\mathrm{d} \Omega} .
$$

A measurement of the reaction $p\left(\vec{\gamma}, \pi^{0}\right) p$ [39] was performed at the Mainz Microtron MAMI [40] using the Glasgow/Mainz tagged photon facility $[1,2]$ and the photon spectrometer TAPS [4]. The MAMI accelerator delivered a continuous wave beam of $405 \mathrm{MeV}$ electrons. Linearly polarized photons were produced via coherent bremsstrahlung in a $100 \mu \mathrm{m}$ thick diamond radiator [41, 42 ] with degrees of polarization of up to $50 \%$. The neutral pion decay photons were detected in TAPS [43], an array of $504 \mathrm{BaF}_{2}$ detectors, which was built up around a liquid-hydrogen target.

The total and differential cross sections were measured over the energy range from $\pi^{0}$ threshold to $168 \mathrm{MeV}$. Figure 7 shows the results for the total cross section in comparison to ref. [33] and [32]. The results for the photon asymmetry are shown in fig. 8 in comparison to the values of ChPT [37] and to a prediction of a dispersion theoretical calculation (DR) by Hanstein, Drechsel and Tiator [44]. The photon asymmetry was determined from all the data between threshold and $166 \mathrm{MeV}$ for which the mean energy was $159.5 \mathrm{MeV}$. The theoretical predictions are shown for the same energy.

The values for the real and imaginary part of $E_{0+}$ and the three $P$-wave combinations were extracted via two multipole fits to the cross sections and the photon asymmetry simultaneously. The two multipole fits differ in the energy dependence of the real parts of the $P$-wave combinations. For the first fit the usual assumption of a behaviour proportional to the product of $q$ and $k$ was adopted ( $q k$-fit, $\chi^{2} /$ dof $\left.=1.28\right)$. The assumption made 
Table 1. Results of both fits ( $q$-fit and $q$-fit) for Re $E_{0+}$ at the $\pi^{0}$ - and $\pi^{+}$-threshold (unit: $10^{-3} / m_{\pi^{+}}$), for the parameter $\beta$ of $\operatorname{Im} E_{0+}$ (unit: $10^{-3} / m_{\pi^{+}}^{2}$ ) and for the three combinations of the $P$-wave amplitudes (unit: $q \cdot 10^{-3} / m_{\pi^{+}}^{2}$ ) with statistical and systematic errors in comparison to the predictions of $\mathrm{ChPT}[37,45]\left(\mathcal{O}\left(p^{3}\right)\right)$ and of a dispersion theoretical approach $(\mathrm{DR},[44])$.

\begin{tabular}{c|c|c|c|c}
\hline & \multicolumn{2}{|c|}{ This work } & ChPT & DR $^{a}$ \\
\hline & $q k$-fit $^{a}$ & $q$-fit & -1.16 & -1.22 \\
\hline$E_{0+}\left(E_{t h r}^{p \pi^{0}}\right)$ & $-1.23 \pm 0.08 \pm 0.03$ & $-1.33 \pm 0.08 \pm 0.03$ & -0.43 & -0.56 \\
$E_{0+}\left(E_{t h r}^{n \pi^{+}}\right)$ & $-0.45 \pm 0.07 \pm 0.02$ & $-0.45 \pm 0.06 \pm 0.02$ & 2.78 & 3.6 \\
$\beta$ & $2.43 \pm 0.28 \pm 1.0$ & $5.2 \pm 0.2 \pm 1.0$ & $9.14 \pm 0.5$ & 9.55 \\
$P_{1}$ & $9.46 \pm 0.05 \pm 0.28$ & $9.47 \pm 0.08 \pm 0.29$ & $-9.7 \pm 0.5$ & -10.37 \\
$P_{2}$ & $-9.5 \pm 0.09 \pm 0.28$ & $-9.46 \pm 0.1 \pm 0.29$ & 10.36 & 9.27 \\
$P_{3}$ & $11.32 \pm 0.11 \pm 0.34$ & $11.48 \pm 0.06 \pm 0.35$ & 11.07 & 9.84 \\
$P_{23}$ & $10.45 \pm 0.07$ & $10.52 \pm 0.06$ & \\
\hline
\end{tabular}

${ }^{a}$ Values of the $P$-wave combinations converted into the unit $q \cdot 10^{-3} / m_{\pi^{+}}^{2}$.

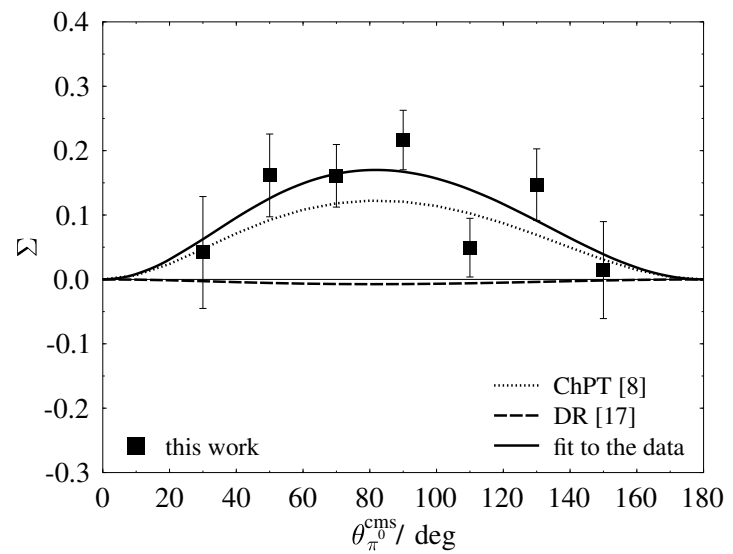

Fig. 8. Photon asymmetry $\Sigma$ for $\pi^{0}$ photoproduction at 159.5 $\mathrm{MeV}$ photon energy with statistical errors (without systematic error of $3 \%$ ) as a function of the polar angle $\theta$ (solid line: fit to the data) in comparison to ChPT [37] (dotted line) and DR [44] (dashed line).

for the second fit is an energy dependence of the $P$-wave amplitudes proportional to $q\left(q\right.$-fit, $\chi^{2} /$ dof $\left.=1.29\right)$. This is the dependence which ChPT predicts for the $P$-wave amplitudes in the near-threshold region, but at higher energies the prediction is in between the $q$ and $q k$ energy dependence.

The results of both multipole fits for Re $E_{0+}$ as a function of the incident photon energy are shown in fig. 9 and compared with the predictions of ChPT and of DR. The results for the threshold values of Re $E_{0+}$ (at the $\pi^{0}$ - and $\pi^{+}$-threshold), for the parameter $\beta$ of $\operatorname{Im} E_{0+}$ and for the values of the threshold slopes of the three $P$-wave combinations of the $q k$-fit and the $q$-fit are summarized in table 1, for more details see [38].

For both fits the low-energy theorems of ChPT $\left(\mathcal{O}\left(p^{3}\right)\right)$ for $P_{1}$ and $P_{2}$ agree with the measured experimental results within their systematic and statistical errors. The experimental value for $P_{3}$ is higher than the value of ChPT, which can be explained by the smaller total and differential cross sections of ref. [32], used by ChPT to determine the dominant low-energy constant $b_{P}$ for this multipole [45]. A new fourth-order calculation in heavy-baryon ChPT by Bernard et al., introduced in [46] and compared

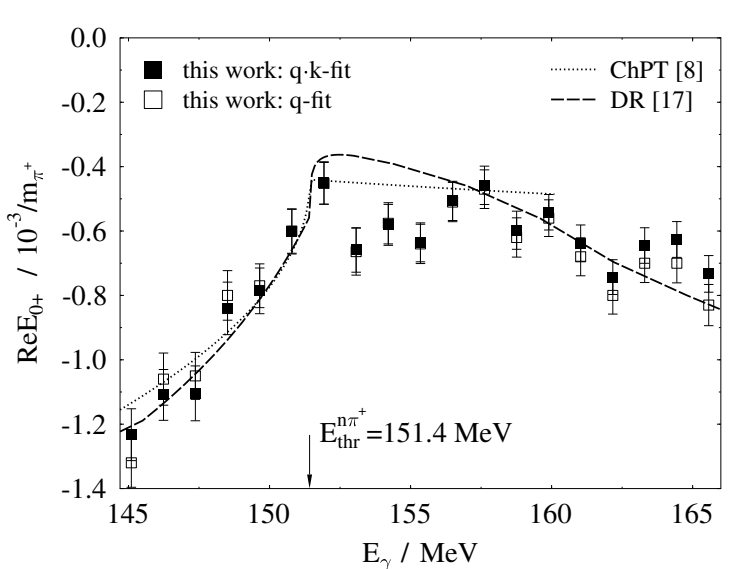

Fig. 9. Results for Re $E_{0+}$ with statistical errors as a function of incident photon energy $E_{\gamma}$ for an assumed energy dependence of the $P$-wave amplitudes proportional to $q \cdot k$ (solid squares) and $q$ (open squares) in comparison to ChPT [37] (dotted line) and DR [44] (dashed line).

to the new MAMI data presented in this letter, shows, that the potentially large $\Delta$-isobar contributions are cancelled by the fourth-order loop corrections to the $P$-wave low-energy theorems. This gives confidence in the thirdorder LET predictions for $P_{1}$ and $P_{2}$, which are in agreement with the present MAMI data. With the new value of $b_{P}$ [46], fitted to the present MAMI data, the ChPT calculation is in agreement with the measured photon asymmetry.

In a recent work, pion photoproduction on the nucleon is evaluated by dispersion relation at constant $t$ [47]. The extension to the unphysical region provides a unique framework to determine the low-energy constants of chiral perturbation theory by global properties of the excitation spectrum. See also the most recent work for pion production at threshold in the framework of covariant baryon chiral perturbation theory [48].

\section{The $\gamma \mathbf{N} \rightarrow \Delta(1232)$ transition and the $\mathrm{E} 2 / \mathrm{M} 1$ ratio}

Low-energy electromagnetic properties of baryons, such as mass, charge radius, magnetic and quadrupole moments 
are important observables for any model of the nucleon structure. In various constituent-quark models a tensor force in the inter-quark hyperfine interaction, introduced first by de Rujula, Georgi and Glashow [49], leads to a $d$-state admixture in the baryon ground-state wavefunction. As a result the tensor force induces a small violation of the Becchi-Morpurgo selection rule [50], that the $\gamma N \rightarrow \Delta(1232)$ excitation is a pure $M 1$ (magnetic dipole) transition, by introducing a non-vanishing E2 (electric quadrupole) amplitude. For chiral quark models or in the Skyrmion picture of the nucleon, the main contribution to the E2 strength stems from tensor correlations between the pion cloud and the quark bag, or meson exchange currents between the quarks. To observe a static deformation ( $d$-state admixture) a target with a spin of at least $3 / 2$ (e.g. $\Delta$ matter) is required. The only realistic alternative is to measure the transition $E 2$ moment in the $\gamma N \rightarrow \Delta$ transition at resonance, or equivalently the $E_{1+}^{3 / 2}$ partial wave amplitude in the $\Delta \rightarrow N \pi$ decay. The experimental quantity of interest to compare with the different nucleon models is the ratio $R_{E M}=E 2 / M 1=E_{1+}^{3 / 2} / M_{1+}^{3 / 2}$ of the electric quadrupole $E 2$ to the magnetic dipole $M 1$ amplitude in the region of the $\Delta(1232)$-resonance. In quark models with $S U(6)$ symmetry, for example the MIT bag model, $R_{E M}=0$ is predicted. Depending on the size of the hyperfine interaction and the bag radius, broken $S U(6)$ symmetry leads to $-2 \%<R_{E M}<0[51,52,53,54]$. Larger negative values in the range $-6 \%<R_{E M}<-2.5 \%$ have been predicted by Skyrme models [55] while results from chiral bag models [56] give values in the range $-2 \%$ to $-3 \%$. The first Lattice QCD result is $R_{E M}=$ $(+3 \pm 9) \%$ [57] and a quark model with exchange currents yields values of about $-3.5 \%$ [58].

The determination of the quadrupole strength $E 2$ in the region of the $\Delta(1232)$ resonance has been the aim of a considerable number of experiments and theoretical activities in the last few years. Experimental results have been published for the differential cross section and photon asymmetry of pion photoproduction off the proton from the Mainz Microtron MAMI and the laser backscattering facility LEGS at Brookhaven National Laboratory, with the results $R_{E M}=-\left(2.5 \pm 0.2_{\text {stat }} \pm 0.2_{\text {sys }}\right) \%$ from the Mainz group [59] and $R_{E M}=-\left(3.0 \pm 0.3_{\text {stat }+ \text { sys }} \pm\right.$ $\left.0.2_{\text {mod }}\right) \%$ from the LEGS group [60]. These new $R_{E M}$ results have started intense discussions about the correct way to extract the $E 2 / M 1$ ratio from the new experimental data. In particular the large variation in the $R_{E M}$ values obtained in theoretical analysis of these data at RPI [61] $\left(R_{E M}=-(3.2 \pm 0.25) \%\right)$, VPI [62] $\left(R_{E M}=-(1.5 \pm 0.5) \%\right)$ and Mainz [63] $\left(R_{E M}=-(2.5 \pm\right.$ $0.1) \%$ ) was quite unsatisfactory. Since small differences in the differential cross section occur in the mentioned MAMI/DAPHNE and LEGS experiments, a new experiment on neutral pion photoproduction off the proton has been performed at the Mainz Microtron covering the full polar angle range of the pion. The new enlarged set of experimental results should allow a determination of $R_{E M}$ more accurately.

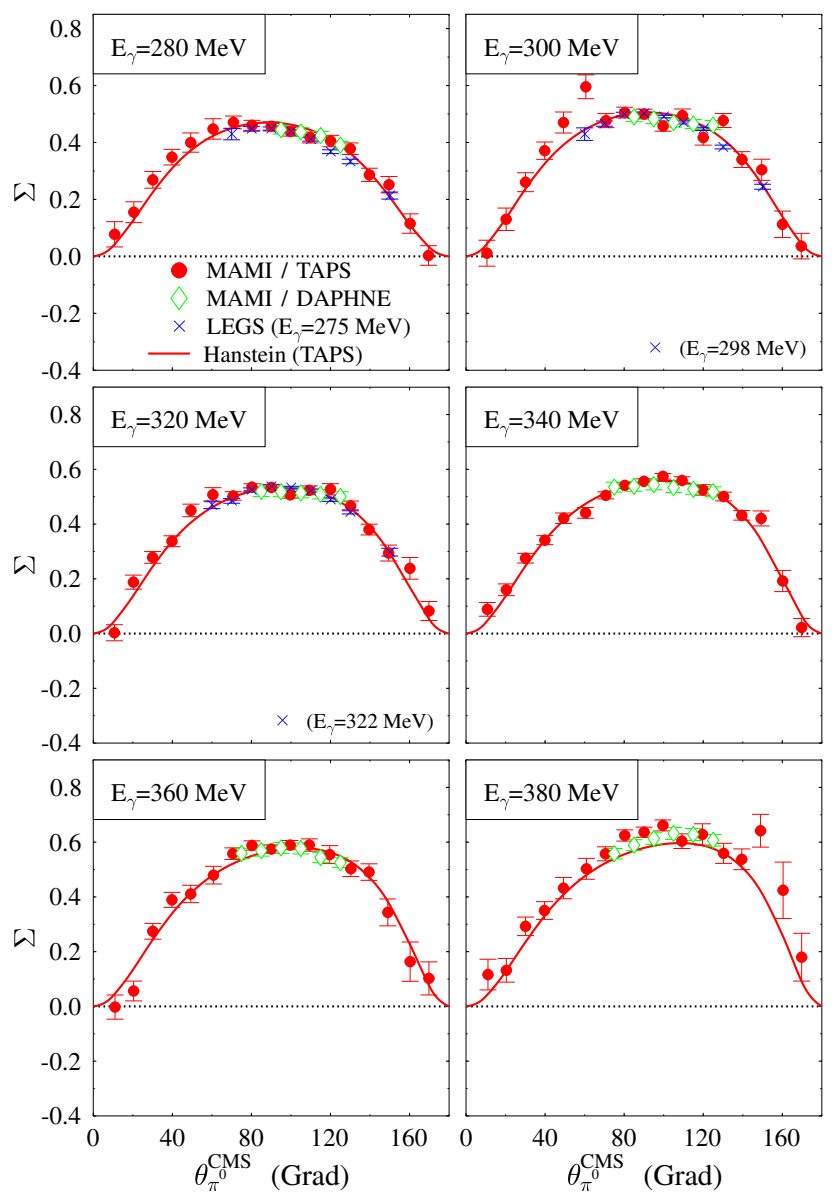

Fig. 10. Photon asymmetries $\Sigma$ in the $\Delta$-resonance region (solid circles, this work ref. [64], open diamonds ref. [59] and crosses ref. [60]).

Figure 10 shows the new results for the photon asymmetry for six different energies in the $\Delta$-resonance region. For the first time this new experiment delivers data in the full polar angle range. The new results are in good agreement with the experimental data of MAMI/DAPHNE and LEGS. In addition, the photon asymmetries of all three experiments are compared to the dispersion theoretical analysis of Hanstein $[63,65]$ and good agreement is found.

The unpolarized differential cross sections for the same six photon energies in the $\Delta$-resonance region are shown in fig. 11. The new results are in agreement with the MAMI/DAPHNE, the LEGS data differ not only in the absolute values of the differential cross section but show as well a different angular distribution. In addition, the results of the Hanstein analysis for the MAMI/TAPS data are shown.

In the angular momentum expansion of the neutral pion photoproduction it is sufficient to take into account $s$ - and $p$-waves, i.e. $l_{\pi}=0$ or 1 only. The angular distributions for the unpolarized cross section $\mathrm{d} \sigma_{0} / \mathrm{d} \Omega$, the parallel part $\mathrm{d} \sigma_{\|} / \mathrm{d} \Omega$ (pion detected in the plane defined by the photon polarization and the photon momentum vector), and perpendicular part $\mathrm{d} \sigma_{\perp} / \mathrm{d} \Omega$ can be expressed in the 

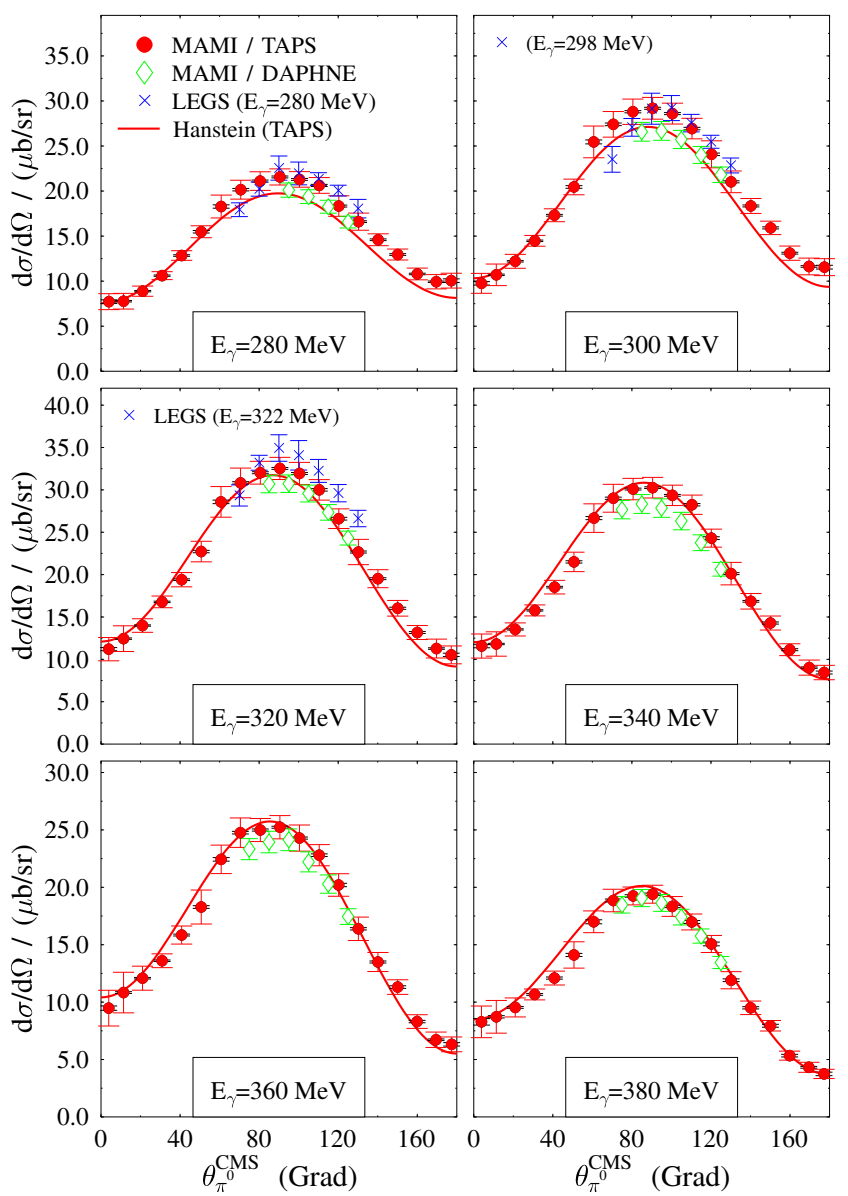

Fig. 11. Differential cross sections in the $\Delta$-resonance region. MAMI/TAPS results are shown with statistical (1-2\%) and systematic errors (solid circles, this work ref. [64], open diamonds ref. [59] and crosses ref. [60]).

$s$ - and $p$-wave approximation by the parameterization

$$
\frac{\mathrm{d} \sigma_{j}(\theta)}{\mathrm{d} \Omega}=\frac{q}{k}\left(A_{j}+B_{j} \cos (\theta)+C_{j} \cos ^{2}(\theta)\right)
$$

where $q$ and $k$ denote the center-of-mass momenta of the pion and the photon, respectively, and $j$ indicates the parallel $(\|)$, perpendicular $(\perp)$ and unpolarized (0) components. The coefficients $A_{j}, B_{j}$ and $C_{j}$ are quadratic or bilinear functions of the $s$ - and $p$-wave amplitudes. In particular, $\mathrm{d} \sigma_{\|} / \mathrm{d} \Omega$ is sensitive to the $E_{1+}$ amplitude, because of interference with $M_{1+}$ in the terms

$$
\begin{aligned}
& A_{\|}=\left|E_{0+}\right|^{2}+\left|3 E_{1+}-M_{1+}+M_{1-}\right|^{2}, \\
& B_{\|}=2 \operatorname{Re}\left[E_{0+}\left(3 E_{1+}+M_{1+}-M_{1-}\right)^{*}\right], \\
& C_{\|}=12 \operatorname{Re}\left[E_{1+}\left(M_{1+}-M_{1-}\right)^{*}\right] .
\end{aligned}
$$

Furthermore, the ratio

$$
R=\frac{1}{12} \frac{C_{\|}}{A_{\|}}=\frac{\operatorname{Re}\left(E_{1+}\left(M_{1+}-M_{1-}\right)^{*}\right)}{\left|E_{0+}\right|^{2}+\left|3 E_{1+}+M_{1+}-M_{1-}\right|^{2}}
$$

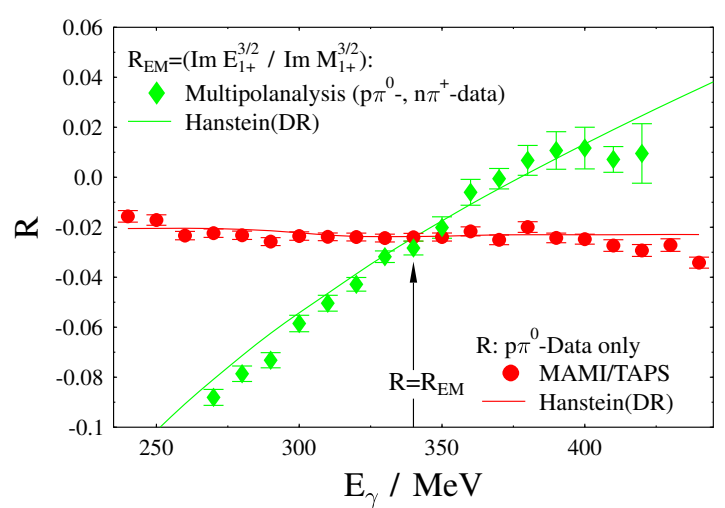

Fig. 12. The energy dependence of the ratio $E_{1+}^{3 / 2} / M_{1+}^{3 / 2}$ is shown as solid diamonds. In addition, the energy dependence of $R=C_{\|} /\left(12 A_{\|}\right)$is shown as solid squares.

can be identified with the ratio $R_{E M}=E_{1+}^{3 / 2} / M_{1+}^{3 / 2}$ at the $\Delta(1232)$-resonance $\left(\delta_{33}=90^{\circ}\right)$

$$
R \simeq R_{E M}=\left.\frac{\operatorname{Im} E_{1+}^{3 / 2}}{\operatorname{Im} M_{1+}^{3 / 2}}\right|_{W=M_{\Delta}} .
$$

This is the crucial point of our analysis [66]. This method offers the advantage of being independent of absolute normalization and insensitive to many systematic errors, because $R_{E M}$ is extracted from the ratio of the coefficients $C_{\|}$and $A_{\|}$fitted to the angular distribution of $\mathrm{d} \sigma_{\|} / \mathrm{d} \Omega$. Further, the following identity can be derived [64]:

$$
R=\frac{1}{12} \frac{C_{\|}}{A_{\|}}=\frac{1}{12} \frac{\frac{C}{A}+\Sigma\left(\theta=90^{\circ}\right)}{1-\Sigma\left(\theta=90^{\circ}\right)} \approx R_{E M},
$$

which depends only on the shape $(C / A)$ of the differential cross section $\mathrm{d} \sigma / \mathrm{d} \Omega$ and the photon asymmetry $\Sigma$ at $\theta^{C M S}=90^{\circ}$. Using eq. (17), the ratio $R_{E M}$ can be extracted [64]:

$$
R_{E M}=\left(-2.4 \pm 0.16_{\text {stat. }} \pm 0.24_{\text {sys }}\right) . \%
$$

According to the Fermi-Watson theorem the $E_{1+}^{3 / 2}$ and $M_{1+}^{3 / 2}$ partial waves have the same phase $\delta_{33}$ and the ratio $E_{1+}^{3 / 2} / M_{1+}^{3 / 2}$ is a real quantity. As shown in fig. 12 , this ratio is strongly dependent on the photon energy and varies from $-8 \%$ at $E_{\gamma}=270 \mathrm{MeV}$ to $+2 \%$ at $E_{\gamma}=420 \mathrm{MeV}$.

\section{Future plans}

The first round of experiments with the Crystal Ball is centered on the first measurement of the magnetic dipole moment of the $\Delta^{+}(1232)$-resonance. The magnetic dipole moment, $\mu_{b}$, provides us with a simple way for testing the validity of the theoretical hadron description in the nonperturbative sector of QCD. This includes quark soliton models, the standard quark models, various effective Lagrangians and lattice QCD calculations. Our experimental 
technique takes advantage of the very short $\Delta$ lifetime by having the $\Delta$ radiatively decay to itself. This method has been successfully pioneered for the $\Delta^{++}$using the reaction $\pi^{+} p \rightarrow \gamma^{\prime} \Delta^{++} \rightarrow \gamma^{\prime} \pi^{+} p$ [67]. We propose to determine $\mu_{b}\left[\Delta^{+}(1232)\right]$ using radiative $\pi^{0}$ photoproduction: $\gamma p \rightarrow \Delta^{+} \rightarrow \gamma^{\prime} \Delta^{+} \rightarrow \gamma^{\prime} \pi^{0} p$. A first pilot experiment $\gamma p \rightarrow \gamma^{\prime} \pi^{0} p$ has been performed with the TAPS calorimeter at MAMI for energies $\sqrt{s}=1221-1331 \mathrm{MeV}$. Angular and energy differential cross section have been determined for all particles in the final state in three bins of the excitation energy [68]. The theoretical aspects have been dealt with in detail already by the theory groups at MAMI [69] and Tuebingen [70]. $\mu_{b}$ can be determined from the differential cross section $\mathrm{d} \sigma^{5} / \mathrm{d} \Omega_{\gamma} \mathrm{d} \Omega_{\pi} \mathrm{d} E_{\gamma}$ and from the asymmetry, $\Sigma$, for linearly polarized photons.

The broad spectrum of MAMI bremsstrahlung photons from $E_{\gamma}^{\text {min }} \approx 100 \mathrm{MeV}$ to $E_{\gamma}^{\max } \approx 1500 \mathrm{MeV}$ together with the $4 \pi$ acceptance of the experimental apparatus allows the simultaneous survey of $\pi^{0}, 2 \pi^{0}, 3 \pi^{0}$ and $\eta$ production at all energies and for the full angular range. Such measurements will be perform with $\mathrm{LH}_{2}$ and $\mathrm{LD}_{2}$ targets using linearly and circularly polarized photon beams. A unique frozen spin target filled with ${ }^{1} \overrightarrow{\mathrm{H}}$, or ${ }^{2} \overrightarrow{\mathrm{H}}$ will be used in the second stage of the experiment (MAMI-C). The target makes possible new high precision, high statistics measurements of the cross sections for the $\vec{\gamma} \vec{N} \rightarrow \pi^{0} N$ and $\vec{\gamma} \vec{N} \rightarrow \pi^{0} \pi^{0} N$ processes at incident photon energies up to $1.5 \mathrm{GeV}$. In particular it provides a unique opportunity to measure the partial contributions to the GDH sum rule on a neutron target in the reactions $\vec{\gamma} \vec{n} \rightarrow \pi^{0} n$ and $\vec{\gamma} \vec{n} \rightarrow \pi^{0} \pi^{0} n$. Our measurements will also provide new information on the photon coupling of lowmass baryon and hyperon resonances. An incomplete list of other possible measurements includes: i) threshold photoproduction of $\pi^{0}$ and $\eta$ at MAMI-B as well as $\eta^{\prime}, \omega$ and $K_{s}^{0}$ at MAMI-C with polarized and unpolarized beams and targets; ii) measurements of the $N^{*}(1535)$ magnetic dipole moment using $\gamma p \rightarrow \gamma^{\prime} \eta p$; iii) a new measurement of the $\eta$ mass.

The new experimental apparatus is shown in fig. 13. The Crystal Ball with TAPS as the forward wall will be used for detection of photons and nucleons. In addition the polar and azimuthal angles of the outgoing proton for $\Theta_{\text {lab }}>20^{\circ}$ will be measured by the central tracker which is based on the DAPHNE cylindrical multiwire proportional chamber. The chamber will be inserted into the Crystal Ball beam cavity.

The Crystal Ball was build at SLAC and used in $J / \psi$ measurements at SPEAR and $b$-quark physics at DESY [71]. The CB is constructed of 672 optically isolated $\mathrm{NaI}(\mathrm{Tl})$ crystals, 15.7 radiation lengths thick. The counters are arranged in a spherical shell with an inner radius of $25.3 \mathrm{~cm}$ and an outer radius of $66.0 \mathrm{~cm}$. The hygroscopic $\mathrm{NaI}$ is housed in two hermetically sealed evacuated hemispheres. Each crystal is shaped like a truncated triangular pyramid, $40.6 \mathrm{~cm}$ high, pointing towards the center of the Ball. The sides on the inner end are $5.1 \mathrm{~cm}$ long and $12.7 \mathrm{~cm}$ on the far end. Electromagnetic showers in

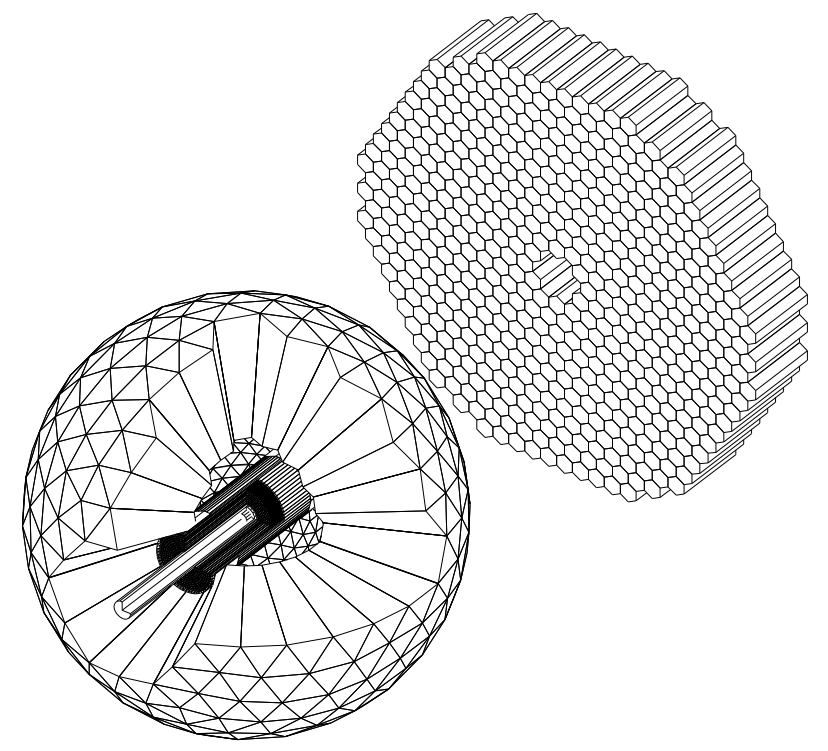

Fig. 13. The Crystal Ball detector and TAPS as forward wall.

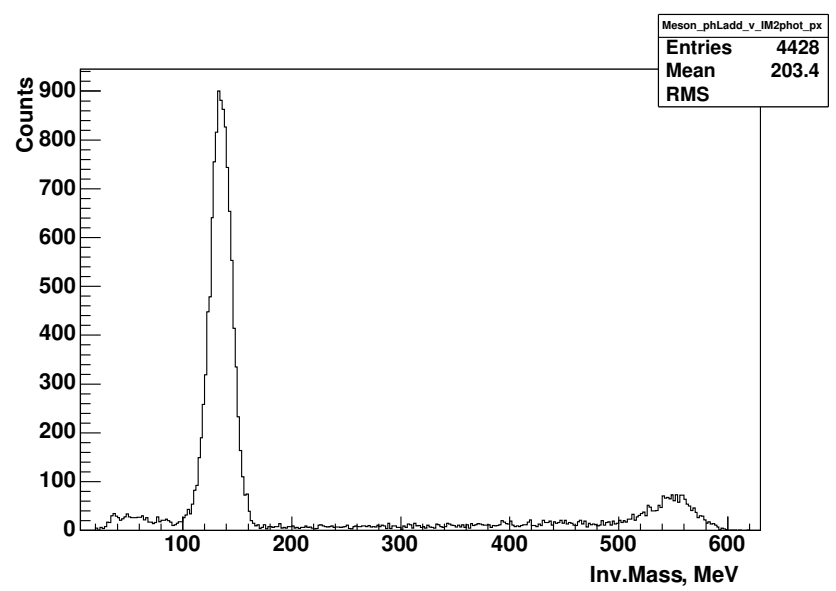

Fig. 14. Invariant mass of two-cluster events for beam photons with energy above $700 \mathrm{MeV}$ after requiring the missing mass to be equal to the mass of proton. The peaks are due to $\pi^{0} \rightarrow 2 \gamma$ and $\eta \rightarrow 2 \gamma$ decays.

the spectrometer are measured with an energy resolution

$$
\sigma_{E} / E \sim 1.7 \% /(E(\mathrm{GeV}))^{0.4}
$$

the angular resolution for photon showers at energies of $0.05-0.5 \mathrm{GeV}$ is $\sigma_{\theta}=2^{\circ}-3^{\circ}$ in the polar angle and $\sigma_{\phi}=$ $2^{\circ} / \sin \theta$ in the azimuthal angle.

High granularity and a large acceptance make the Crystal Ball a unique instrument for measuring reactions with multiphoton final states. The $\mathrm{CB}$ detects neutrons with an efficiency of $\approx 35 \%$ at $E_{n}=150 \mathrm{MeV}$ [72].

The first production run of the CB@MAMI program, a measurement of the photon asymmetry in $\pi^{0}$ photoproduction at threshold, was accomplished in July-August 2004. In October 2004 we have started a 600 hours long production run for the measurements of the $\Delta^{+}(1232)$ magnetic dipole moment. Figures $14-17$ illustrate the 


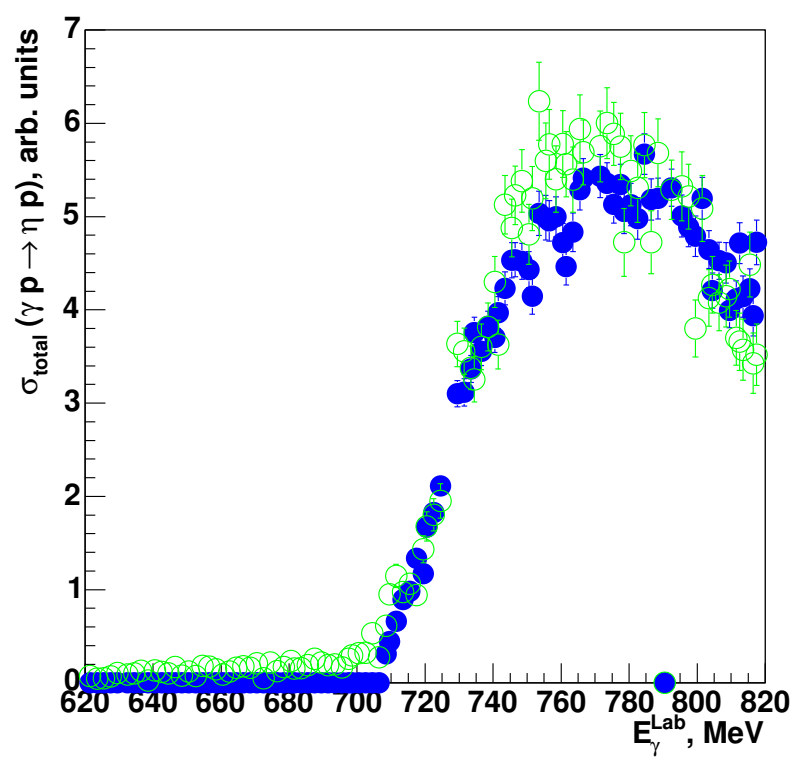

Fig. 15. The total cross section of $\gamma p \rightarrow \eta p$ in arbitrary units from $\eta \rightarrow 2 \gamma$ decay modes (solid circles) is compared to the $\gamma p \rightarrow 3 \pi^{0} p$ total cross section (open circles). Above the $\eta$ threshold the $\gamma p \rightarrow 3 \pi^{0} p$ is mainly from $\eta \rightarrow 3 \pi^{0}$ decay. The total cross sections are relatively normalized at $E_{\gamma}=730 \mathrm{MeV}$.

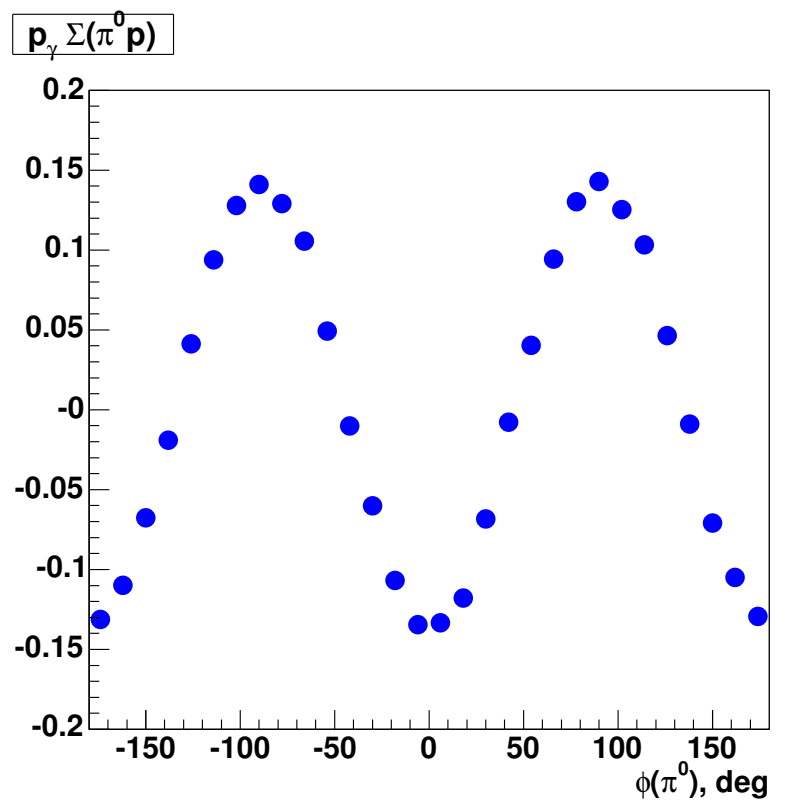

Fig. 16. $\phi$-dependence of the beam photon asymmetry times polarization, $p_{\gamma} \times \Sigma(\phi)$, for the reaction $\gamma p \rightarrow \pi^{0} p$, where $\Sigma(\phi)=\frac{\sigma_{\|}(\phi)-\sigma_{\perp}(\phi)}{\sigma_{\|}(\phi)+\sigma_{\perp}(\phi)}$. The function shows clear $\cos (2 \phi)$ behavior over the full angular range. The data covers the beam energy interval of $360-450 \mathrm{MeV}$ and is integrated over $\Theta_{\pi^{0}}$.

quality of the data, showing some characteristic distributions.

The invariant mass of two photons for an incident beam with energy above $700 \mathrm{MeV}$ is shown in fig. 14. The two peaks of the spectra are due to the reactions $\gamma p \rightarrow$ $\pi^{0}(\gamma \gamma) p$ and $\gamma p \rightarrow \eta(\gamma \gamma) p$. The two-gamma invariant mass is shown for events with the missing mass equal to

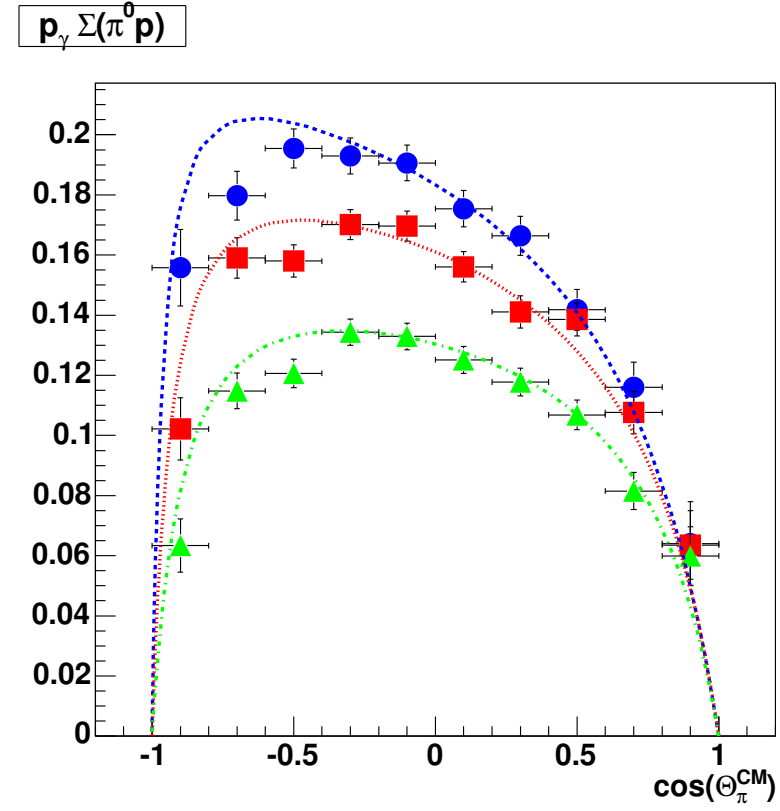

Fig. 17. Preliminary results for the photon asymmetry times beam polarization shown as a function of $\cos \Theta_{\pi^{0}}$ in c. m. for $E_{\gamma}=375 \mathrm{MeV}$ (triangles), $405 \mathrm{MeV}$ (squares), and $436 \mathrm{MeV}$ (circles), compared to MAID predictions. The MAID curves are normalized to the experimental data at $\cos \Theta_{\pi}=0$.

the mass of the proton. Figure 15 shows an excitation function for $\gamma p \rightarrow \eta(\gamma \gamma) p$ in arbitrary units. The total cross section for $\gamma p \rightarrow 3 \pi^{0} p$ is shown on the same figure for comparison. Below $\eta$ threshold $3 \pi^{0}$ events are produced via sequential decay of resonances, while above the $\eta$-threshold most of the events are produced by the $\eta \rightarrow 3 \pi^{0}$ decay.

The experimental setup made up of the Crystal Ball and TAPS is almost perfectly $\phi$-symmetric. Together with the good quality polarized MAMI beam it allows high statistics, low systematics uncertainty, polarization measurements. Figure 16 shows the $\phi$-dependence of the beam photon asymmetry, $\Sigma(\phi)$, for the reaction $\gamma p \rightarrow \pi^{0} p$. The asymmetry is not corrected for the beam polarization. The data represent about $5 \%$ of the statistics obtained in the course of our most recent $\mu(\Delta(1232))$ run. The photon asymmetry as a function of $\cos \Theta_{\pi^{0}}$ is shown in fig. 17 for beam photon energies of $375 \mathrm{MeV}, 405 \mathrm{MeV}$, and $436 \mathrm{MeV}$ in comparison with MAID [73]. The results are very preliminary. The measured distributions are not corrected for the beam photon polarization therefore the MAID curves are normalized to the data at $\cos \Theta_{\pi}=0$. The photon asymmetries obtained in our experiment show good agreement with the MAID evaluations for $\cos \Theta_{\pi}>0$ and slightly deviate in the backward angles. The difference between our data and MAID gets larger at higher beam energies.

I would like to thank the organizers Hartmuth Arenhövel, Hartmut Backe, Dieter Drechsel, Karl-Heinz Kaiser and Thomas Walcher of the symposium "20 Years of Physics at the Mainz Microtron MAMI". 


\section{References}

1. I. Anthony et al., Nucl. Instrum. Methods A 301, 230 (1991).

2. S.J. Hall et al., Nucl. Instrum. Methods A 368, 698 (1996).

3. URL: http://wwwa2.kph.uni-mainz.de/A2.

4. R. Novotny, IEEE Trans. Nucl. Sci. 38, 379 (1991).

5. G. Audit et al., Nucl. Instrum. Methods A 301, 473 (1991).

6. A. Partridge et al., Paper presented to the IEEE Meeting, San Francisco, October 1977 (Stanford Linear Accelerator Center, Stanford, 1977).

7. V.A. Petrun'kin, Sov. Phys. JETP 13, 808 (1961).

8. V.A. Petrun'kin, Sov. J. Part. Nucl. 12, 278 (1981).

9. V. Olmos de León, PhD Thesis, Mainz University (2000).

10. V. Olmos de León et al., Eur. Phys. J. A 10, 207 (2001).

11. A. L'vov et al., Sov. J. Nucl. Phys. 34, 597 (1981).

12. A. Zieger et al., Phys. Lett. B 278, 34 (1992).

13. J.F. Donoghue, B.R. Holstein, Phys. Rev. D 40, 2378 (1989); J. Bijnens, F. Cornet, Nucl. Phys. B 296, 557 (1988); B.R. Holstein, Comments Nucl. Part. Phys. 19, 221 (1990); S. Belluci, J. Gasser, M.E. Sainio, Nucl. Phys. B 423, 80 (1994).

14. U. Bürgi, Nucl. Phys. B 79, 392 (1997).

15. A.N. Ivanov, M. Nagu, N.I. Troitskaya, Mod. Phys. Lett. A 7, 1997 (1992).

16. L.V. Fil'kov, I. Guiasu, E.E. Radescu, Phys. Rev. D 26, 3146 (1982).

17. L.V. Fil'kov, V.L. Kashevarov, Eur. Phys. J. A 5, 285 (1999).

18. V. Bernard, B. Hiller, W. Weise, Phys. Lett. B 205, 16 (1988).

19. M.A. Ivanov, T. Mizutani, Phys. Rev. D 45, 1580 (1992).

20. Yu.M. Antipov et al., Phys. Lett. B 121, 445 (1983).

21. T.A. Aybergenov et al., Sov. Phys. Lebedev Inst. Rep. 6, 32 (1984); Czech. J. Phys. B 36, 948 (1986).

22. J. Portolés, M.R. Pennington, The Second DA $\phi N E$ Physics Handbook, Vol. 2 (1995) p. 579, hep-ph/9407295.

23. J. Boyer et al., Phys. Rev. D 42, 1350 (1990).

24. F. Donoghue, B. Holstein, Phys. Rev. D 48, 137 (1993).

25. T. Walcher, Prog. Part. Nucl. Phys. 24, 189 (1990).

26. J. Ahrens et al., Nucl. Phys. News 4, 5 (1994).

27. P. Grabmayer et al., Nucl. Instrum. Methods A 402, 85 (1998).

28. Ch. Unkmeir, PhD Thesis, Mainz University (2000).

29. J. Ahrens et al., Eur. Phys. J. A 23, 113 (2005).

30. E. Mazzucato et al., Phys. Rev. Lett. 57, 3144 (1986).

31. R. Beck et al., Phys. Rev. Lett. 65, 1841 (1990).

32. M. Fuchs et al., Phys. Lett. B 368, 20 (1996).

33. J.C. Bergstrom et al., Phys. Rev. C 53, R1052 (1996); 55, 2016 (1997)

34. P. de Baenst, Nucl. Phys. B 24, 633 (1970).

35. I.A. Vainshtein, V.I. Zakharov, Nucl. Phys. B 36, 589 (1972).

36. V. Bernard, J. Gasser, N. Kaiser, U.-G. Meißner, Phys. Lett. B 268, 291 (1991)
37. V. Bernard, N. Kaiser, U.-G. Meißner, Z. Phys. C 70, 483 (1996).

38. A. Schmidt, Phys. Rev. Lett. 87, 232501 (2001).

39. A. Schmidt, Doktorarbeit, University Mainz (2001).

40. H. Herminghaus, K.H. Kaiser, H. Euteneuer, Nucl. Instrum. Methods A 138, 1 (1976).

41. D. Lohmann, J. Peise et al., Nucl. Instrum. Methods A 343, 494 (1994).

42. A. Schmidt, Diplomarbeit, University Mainz (1995).

43. R. Novotny, IEEE Trans. Nucl. Sci. 43, 1260 (1996).

44. O. Hanstein, D. Drechsel, L. Tiator, Phys. Lett. B 399, 13 (1997).

45. V. Bernard, N. Kaiser, U.-G. Meißner, Phys. Lett. B 378 , 337 (1996).

46. V. Bernard, N. Kaiser, U.-G. Meißner, Eur. Phys. J. A 11, 209 (2001).

47. B. Pasquini, D. Drechsel, L. Tiator, Eur. Phys. J. A 23, 279 (2005)

48. V. Bernard, B. Kubis, U.-G. Meißner, arXiv:nucl-th/ 0506023v1 (2005).

49. A. Rujula, H. Georgi, S.L. Glashow, Phys. Rev. D 12, 147 (1975).

50. C. Becchi, G. Morpurgo, Phys. Lett. 17, 352 (1965).

51. R. Koniuk, N. Isgur, Phys. Rev. D 21, 1868 (1980).

52. S.S. Gershteyn et al., Sov. J. Nucl. Phys. 34, 870 (1981).

53. D. Drechsel, M.M. Giannini, Phys. Lett. B 143, 329 (1984).

54. S. Capstick, Phys. Rev. D 46, 2864 (1992).

55. A. Wirzba, W. Weise, Phys. Lett. B 188, 6 (1987).

56. K. Bermuth et al., Phys. Rev. D 37, 89 (1988).

57. D.B. Leinweber, Proceedings of the International Conference "Baryons92" (1992) p. 29.

58. A. Buchmann et al., Phys. Rev. C 55, 448 (1997).

59. R. Beck, H.-P. Krahn et al., Phys. Rev. Lett. 78, 606 (1997).

60. G.S. Blanpied et al., Phys. Rev. Lett. 79, 4337 (1997).

61. R.M. Davidson, N.C. Mukhopadhyay, Phys. Rev. Lett. 79, 4509 (1997).

62. G. Keaton, R.L. Workman, Phys. Rev. Lett. 79, 4511 (1997).

63. O. Hanstein et al., Phys. Lett. B 385, 45 (1996).

64. R. Leukel, PhD Thesis, University Mainz, 2001.

65. O. Hanstein et al., Nucl. Phys. A 632, 561 (1998).

66. R. Beck et al., Phys. Rev. C 61, 035204 (2000).

67. B. Nefkens et al., Phys. Rev. D 18, 3911 (1978).

68. M. Kotulla et al., Phys. Rev. Lett. 89, 272001 (2002).

69. D. Drechsel, M. Vanderhaeghen, Phys. Rev. C 64, 065202 (2001).

70. A.I. Machavariani, A. Faessler, arXiv:nucl-th/0202060 (2002).

71. E.D. Bloom, C.W. Peck, Annu. Rev. Nucl. Sci. 33, 143 (1983).

72. T.D. Stanislaus et al., Nucl. Instrum. Methods A 462, 12 (2001).

73. D. Drechsel et al., Nucl. Phys. A 645, 145 (1999). 